\title{
Optimization of construction of a shell for launcher in the water medium in the supercavitation regime
}

\author{
Vladimir Arkhipov ${ }^{1}$, Nikolay Zolotorev ${ }^{1}$, and Ksenia Perfilieva ${ }^{1, *}$ \\ ${ }^{1}$ National Research Tomsk State University, 634050 Tomsk, Russia
}

\begin{abstract}
New scheme of a shell, providing increase in range and stability of the movement in the water medium due to realization of the supercavitation regime on all trajectory of its movement, has been considered. The results of the analysis in the efficiency of the movement of a shell in the water environment in the supercavitation mode are presented.
\end{abstract}

\section{Introduction}

When the shell moves in the water medium, the resistance force increases sharply, since the water density is three orders of magnitude higher than the air density [1]. To increase the firing range and ensure sufficient speed of the shell at the end point of the trajectory, it is necessary to increase the initial speed of the $V_{0}$ shell, reduce the area of the mid-beam section of the $S_{M}$ and reduce the resistance coefficient of the $C_{x}$. Increasing the muzzle velocity $V_{0}$ is inefficient because it increases the strength of the resistance. As the caliber of the shell decreases, its mass and, consequently, the initial kinetic energy decreases, which leads to a decrease in the firing range. A significant reduction in the resistance coefficient can be obtained when the solid moves in the super-cavitation mode [2]. In this case, a cavity filled with water vapor is formed around the solid with a special head cavitator.

In this paper, the scheme of the shell for firing in an aqueous medium was considered, which allows to increase the range of motion of the target element in the aqueous medium and increase the stability of its motion.

\section{The scheme of the shell for launcher in the water}

To increase the range and stability of movement in the water environment, a new scheme of the shell [3] is proposed (Fig. 1). Due to the motion of the shell in the barrel of the weapon, ignition of the end surface of the retarder 16 occurs. The decelerator 16 , prevents the ignition of the solid fuel charge 2 by the combustion products of the propellant charge. After the shell leaves the barrel of the gun with an initial velocity $V_{0}$, its movement takes place in the water medium in the super-cavitation mode. After a certain period of time $t *$, the velocity of the shell is reduced to a critical value $V_{*}$, at which the super-cavitation mode

\footnotetext{
* Corresponding author: k.g.perfiljeva@yandex.ru
} 
is not implemented. At the time $t_{i g n}=t *$ the retarder 16 completely burns, and the combustion products of the retarder initiates the pyrotechnic igniter 6 which is fixed on it. The products of combustion of the igniter 6 are received in the through axial channel 7 of the solid propellant charge 2 and burns it. The combustion products of the solid fuel charge 2 enter through the through axial channel 7 into the communicating through channel 10 of the ballistic tip 3 and push the cylindrical plug 14 out of the contraction of the 11 through channel 10. In this case, the combustion products of the solid fuel charge 2 flow into the aqueous medium through the Central nozzle 5 and through the narrowing of the 14 channel 10. Part of the combustion products through the radial channels 15 enter the annular gap 9 and flow into the water medium through the annular nozzle 13 at an angle $\beta$ to the longitudinal axis of the shell in the direction of its stern.

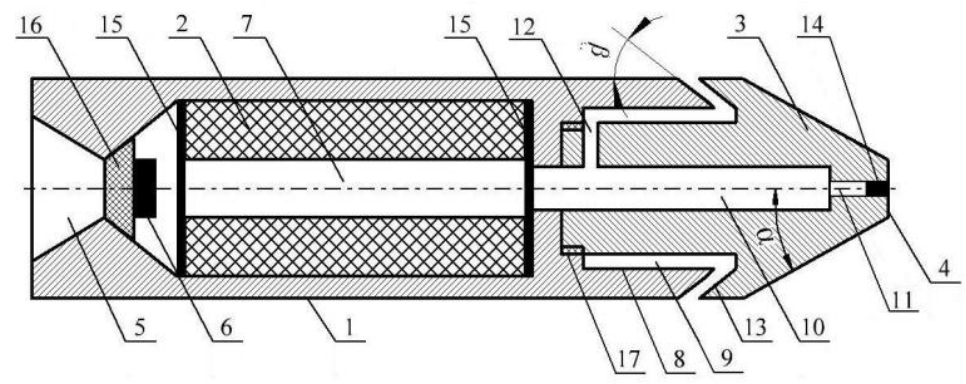

Fig. 1. Scheme of the shell to fire in the water environment.

Thus, the additional injection of the combustion products of the solid fuel charge 2 through the narrowing 11 and the annular nozzle 13 ensures the preservation of the superpressure mode at the velocities of the shell $V<V_{*}$. In this case, the flow of combustion products through the Central nozzle 5 and the annular nozzle 13 creates an additional reactive force, which increases the speed and range of motion of the shell.

\section{Analysis of the effectiveness of the shell motion in the water environment in the supercavitation mode}

Consider the movement of the shell caliber $0.03 \mathrm{~m}$ in the water environment at a depth of $H$ $=20 \mathrm{~m}$ with an initial (muzzle) velocity $V_{0}=300 \mathrm{~m} / \mathrm{s}$.

The hydrostatic pressure at this depth is:

$$
p(H)=10^{5}(1+0.1 H) .
$$

The choice of the critical value of the shell velocity $V_{*}$ at which the solid fuel charge is ignited is determined by the following factors. The main criterion for the super-cavitation motion of a shell in an aqueous medium is the number of cavitation [4]:

$$
\sigma=\frac{2\left[p(H)-p_{0}\right]}{\rho V^{2}},
$$

where $p_{0}=2336.8 \mathrm{~Pa}$ is the pressure of saturated water vapor during bubble cavitation.

The super-cavitation mode is realized at values $\sigma<\sigma_{*}=0.06$ [4]. From the formula (2) the ratio for the velocity of the shell follows, which provides its motion in the supercavitation mode: 


$$
V \geq V_{*}=\sqrt{\frac{2\left[p(H)-p_{0}\right]}{\rho \cdot \sigma_{*}}} .
$$

Thus, the value depends on the depth of immersion $H$, where the shell moves [5]. Map modes of movement of the shell in the coordinates $(H, V)$ is shown in Fig. 2. The line that defines the boundary of the supercavitation region corresponds to the value $\sigma=\sigma_{*}=0.06$.

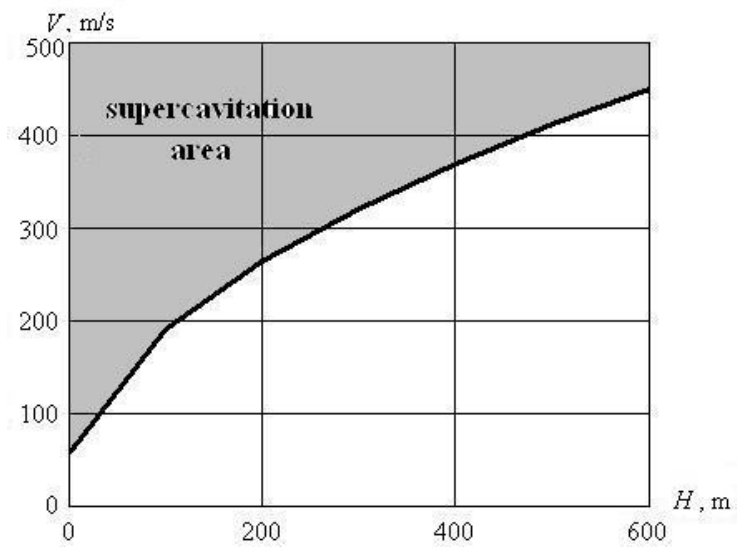

Fig. 2. Map modes of movement of the shell in the coordinates $(H, V)$.

Choose a solid fuel charge with a diameter $D=0.026 \mathrm{~m}$ and a length $l=0.2 \mathrm{~m}$. Set the charge channel in the form of a star, then the combustion area will correspond to the area of the cylindrical channel with a diameter $d=0.013 \mathrm{~m}$.

As a solid fuel, we choose a mixture fuel TPQ-3011A, the characteristics of which are given in Table 1.

Table 1.Characteristics of fuel mixture TP-Q-3011A [6].

\begin{tabular}{|c|c|c|c|c|c|c|}
\hline Parameter & $\boldsymbol{P}_{\boldsymbol{f}}, \mathbf{k g} / \mathbf{M}^{\mathbf{3}}$ & $\boldsymbol{u}_{\mathbf{1}}, \mathbf{m} / \mathbf{s}$ & $\mathbf{v}$ & $\boldsymbol{k}$ & $R, \mathbf{J} / \mathbf{K g} \cdot \mathbf{K})$ & $\boldsymbol{T}_{\boldsymbol{p}}, \mathbf{K}$ \\
\hline Value & 1630 & $0.69 \cdot 10^{3}$ & 0.32 & 1.26 & 378 & 1260 \\
\hline
\end{tabular}

The main characteristics of the solid-fuel engine, obtained by the results of the intraballistic calculation of the SRM, carried out by the method [6] are given in Table 2 .

Table 2. Characteristics of the solid-fuel engine of the shell.

\begin{tabular}{|c|c|c|c|c|}
\hline Parameter & $\boldsymbol{P}, \mathbf{N}$ & $\boldsymbol{G}, \mathbf{~ k g} / \mathbf{s}$ & $\boldsymbol{u}, \mathbf{m} / \mathbf{s}$ & $\boldsymbol{t}_{\boldsymbol{c}}, \mathbf{s}$ \\
\hline Narrowing of the nozzle & 17.4 & 0.02 & 870 & \multirow{2}{*}{2.145} \\
\hline Nozzle assembly & 23.5 & 0.01 & 2349 & \\
\hline
\end{tabular}

Consider the characteristics of the motion of models in an aqueous medium with an initial velocity $V_{0}=300 \mathrm{~m} / \mathrm{s}$., $V_{*}=100 \mathrm{~m} / \mathrm{s}$. The calculations of the inert shell (M1) and the shell with rdtt (M2). The main parameters of the models are shown in Table 3.

Table 3. Model parameters.

\begin{tabular}{|c|c|c|c|c|c|}
\hline Model & $\boldsymbol{m}, \mathbf{k g}$ & $\boldsymbol{\rho}, \mathbf{~ k g} / \mathbf{m}^{\mathbf{3}}$ & $\boldsymbol{D}_{\boldsymbol{k}}, \mathbf{m}$ & $\boldsymbol{D}_{\boldsymbol{n}}, \mathbf{m}$ & $\boldsymbol{C}_{\boldsymbol{x}}$ \\
\cline { 1 - 3 } $\mathrm{M} 1$ & 1 & $10^{3}$ & $5 \cdot 10^{-3}$ & $30 \cdot 10^{-3}$ & 0.82 \\
\cline { 1 - 2 } & 1 & & & & 0.46 \\
\hline
\end{tabular}


When the model moves along the trajectory, two sections are realized. In the first section the model moves with the working SRM, in the second section the model moves as an inert body.

The classical equation of motion of a body of constant mass $m$ in a continuous medium has the form:

$$
m \frac{d V}{d t}=-C_{x} S_{M} \frac{\rho V^{2}}{2},
$$

where $m$ - the mass of the model; $C_{x}$ - dimensionless coefficient of resistance; $S_{M}-$ the area of the mid-body section.

The main criterion of super-cavitation motion of the shell in the water medium is the number of cavitation (2). Evaluation [7-8] show that when the values of the cavitation $\sigma<\sigma_{*}=0.06$ super-cavitation model experiences less resistance than the same model with the continuous flow. At high speeds and low depths of immersion $\sigma<<1$, and therefore $C_{x}=0.82$ the equation of motion (6) has the form:

$$
\frac{d V}{d t}=-k V^{2},
$$

where $k=C_{x} \frac{\rho S_{k}}{2 m}=$ const .

Integrating equation (7) (with $u=u_{0}$ for $t=0$ ), we obtain a formula for the dependence of the velocity of the inert model on time:

$$
V(t)=\frac{V_{0}}{1+k V_{0} t}
$$

When the model moves on the active part of the trajectory, the equation of motion of the model (6) changes taking into account the thrust of the rocket engine and has the following form

$$
m \frac{d V}{d t}=P-C_{x} S_{k} \frac{\rho V^{2}}{2}
$$

where $P$ is the thrust force.

The equation of motion (9) is presented as

$$
\frac{d u}{d t}=-k u^{2}+a
$$

where $a=\frac{P}{m}=$ const .

Integrating equation (10), we obtain an algebraic formula for calculating the speed of the model with the working SRM from time

$$
u(t)=A \frac{\left(C+e^{-B \cdot t}\right)}{\left(C-e^{-B \cdot t}\right)},
$$

The distance traveled by the models over time is determined by the equation 


$$
S=u \cdot t
$$

The ignition delay time $t_{i g n}$ is chosen to be equal to the time $t *$, during which the shell speed will decrease to the value $V_{*}$

$$
V_{*}=\frac{V_{0}}{1+k V_{0} t_{*}},
$$

From (13) the ratio follows to determine the ignition delay time of the solid fuel charge which is determined by the ratio

$$
t_{\text {ign }}=t_{*}=\frac{1}{k}\left(\frac{V_{0}}{V_{*}}-1\right)=\frac{2 m_{0}}{C_{x} \rho V_{0} S_{k}}\left(\frac{V_{0}}{V_{*}}-1\right),
$$

The results of parametric calculations of the motion of models in the water environment are given in Table 4.

Table 4. The results of parametric calculations of the motion of models in the water environment.

\begin{tabular}{|c|c|c|c|c|}
\hline Model & $\boldsymbol{t} * \mathbf{S}$ & $\boldsymbol{V}_{*}, \mathbf{m} / \mathbf{s}$ & $\boldsymbol{L}_{*}^{*} \mathbf{m}$ & $\boldsymbol{L}_{\boldsymbol{f}}, \mathbf{m}$ \\
\hline M1 & \multirow{2}{*}{0.82} & \multirow{2}{*}{100} & \multirow{2}{*}{131.08} & 136 \\
\cline { 1 - 3 } \cline { 4 - 5 } & & & 305 \\
\hline
\end{tabular}

The calculated dependences of the speed of the models on time are shown in Fig. 3, and the distances covered by the models are shown in Fig. 4, where the inert shell (M1), missiles with SRM (M2).

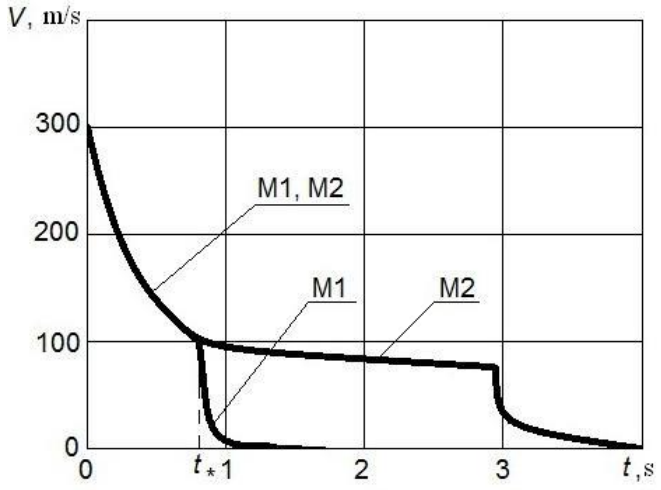

Fig. 3. The estimated velocity models M1, M2.

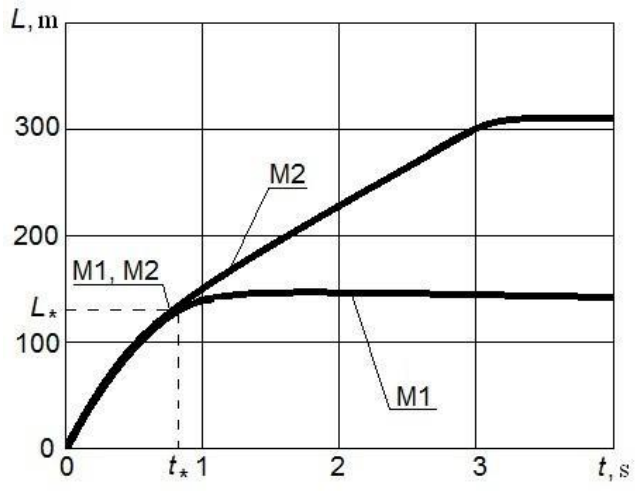

Fig. 4. The distance covered by the models M1, M2. 


\section{Conclusion}

- A modified scheme of the shell for firing in the aquatic environment, was presented, which allows to increase the range of motion in throwing the item in the aquatic environment and increase the sustainability of its movement due to the provision of the mode of supercavitation in all its trajectory.

- The analysis of the dependence of the shell velocity on the depth of immersion was carried out and the boundary of the supercavitation area was determined.

- Certain parameters of solid fuel engine were determined, ensuring the preservation of the regime of supercavitation at speeds of projectile motion $V<V$ *

- External ballistic series of calculations of the motion of inert shell (M1) and shell with solid propellant motors (M2) in the aquatic environment were determined.

- It is determined that for the new shell scheme when moving in the water environment in the super-pressure mode, the speed and the distance traveled far exceed the corresponding values when moving the model without a cavitator.

This research was supported by "The Tomsk State University competitiveness improvement programe"

\section{References}

1. M. E. Deich, Technical gas dynamics (Moscow, Gosenergoizdat, 1961) [in Russian]

2. Yu. D. Vlasenko, Applied hydro mechanics 2, 26 (2000) [in Russian]

3. V. A. Arkhipov, A. I. Konovalenko, K. G. Perfilieva, Shell for launcher in the water medium. The application on patent RU 2017135869, (Int.Cl.: F42B 5/02, 2017) [in Russian]

4. Yu. N. Savchenko, A. N. Swarovski, Appl. fluid mech. 11, 69 (2009) [in Russian]

5. G. Thomas, Torpedo body form and gas layer control. Patent US No. 3205846, (Int.Cl.: F42B 19/12, 1965)

6. A. A. Shishkov, S. D. Panin, B. V. Rumyantsev, Working processes in rocket engines solid fuels (Moscow, Machine Building, 1988) [in Russian]

7. Yu. N. Savchenko, V. N. Semenenko, S. I. Putilin, Appl. fluid mech. 1, 79 (1999) [in Russian]

8. Yu. N. Savchenko, G. Yu. Savchenko, Appl. fluid mech. 6, 78 (2004) [in Russian] 